\title{
La geometría en el álgebra de los números reales
}

fecha de recepción: 20/11/2019 • Fecha de aceptación: 03/01/2020 • Fecha de ublicación:10/02/2020

Mauro Javier Mendizabal Freire Universidad Tecnológica Israel mmendizabal@uisrael.edu.ec https://orcid.org/0000-0001-8209-4351

Mauro Javier Mendizabal Pico Pontifica Universidad Católica de Chile murogrande@yahoo.com https://orcid.org/0000-0002-7150-2708

\section{RESUMEN}

Este documento expresa como la representación escrita en forma analítica de un teorema del conjunto de los números reales, acompañada de un gráfico geométrico, confirma la veracidad y validez de la demostración además de mostrarla de una manera visual.

PALABRAS CLAVE: representaciones, gráficas, geométricas, visuales, números reales.

\section{ABSTRACT}

This document expresses how the writting representation in analytical form of a theorem in the set of real numbers, accompanied by a geometric graph, confirms the truthfulness and validity of the proof showing it in a visual way too.

KEYWORDS: representations; graphical; geometric; visual; real numbers. 


\section{INTRODUCCIÓN}

La Matemática es un edificio que, en sus inicios, fue construido por el techo y no por su base o cimientos. Esta forma de construcción aseguraba que la estructura colapsara en cualquier momento. A medida que la Matemática fue desarrollándose, surgió la necesidad de dotar a este gran edificio del cemento y concreto necesarios que garantizaran la permanencia de la estructura en pie, Alba (1938). Es así como Bertrand Russell (1872-1970) incorpora a la Matemática la lógica aristotélica con el método deductivo de demostración y las reglas de deducción o inferencia. Es decir, la Matemática, una ciencia eminentemente deductiva y formal, que está a nivel de la Lógica y la Filosofía, tiene en la demostración lo que hacía falta para ser el edificio de conocimientos exactos más esplendoroso que la mente humana seguirá construyendo.

La enseñanza y aprendizaje de la Matemática conllevan la realización de demostraciones, Oliveros (2002). Cuando se imparte una clase de Matemática y se demuestran teoremas, se usan definiciones, axiomas o postulados y otros teoremas previamente demostrados y aceptados como proposiciones verdaderas, Díaz (2014). El razonamiento o silogismo, así elaborado, constituye la demostración analítica formal algébrica del teorema. Inferencias como la inducción completa pueden considerarse como otro tipo de demostración.

En una clase de Matemática, no solo se resuelven problemas y se desarrollan ejercicios, sino que se busca demostrar teoremas aplicando la deducción o el método deductivo de demostración. Antes de demostrar un teorema, se piensa en la secuencia o cadena de pasos lógicos a seguir en el razonamiento. Si a este proceso, se acompaña con las respectivas justificaciones y se añade un gráfico; se ilustra, muestra y visualiza aquello que se desea demostrar.

Desarrollar ejercicios o resolver problemas en Matemática sirve, no solo, para afirmar la comprensión y aplicabilidad del teorema que se ha demostrado, sino también para que los estudiantes ejerciten acciones que exige la solución del ejercicio o problema.

Cuando un docente trata un nuevo teorema se enfrenta a dos situaciones. La primera, enunciar el teorema sin demostrarlo porque resulta tedioso y demasiado aburrido para los estudiantes. Y la segunda, enunciar el teorema y demostrarlo.

Si el docente elige la primera situación, está perdiendo la oportunidad que los estudiantes desarrollen la abstracción y la capacidad de deducir. Si escoge la segunda, sabe con anticipación, que muchos estudiantes protestarán o simplemente dejarán de poner atención a la demostración porque rechazan los procesos donde el pensar se hace necesario, Oliveros (2002).

En la antigua Grecia, eran instrumentos indispensables para hacer Geometría, y por ende Matemáticas; la escuadra, la regla y el compás. Todo lo que se hacía con números tenía que ser medido, construido y mostrado para que tuviera validez. Se llegó al caso, incluso, de que la escuela pitagórica cerró cuando no se pudo mostrar el triángulo rectángulo, cuyas medidas de construcción, dieran el número exacto de gnómodas para la hipotenusa si los catetos medían valores iguales. Por tanto, mostrar o visualizar ya desde esa época pasó a ser tan importante como ahora para aprender y entender Matemáticas. 
Si relacionamos la forma como en la Grecia Clásica se mostraba la validez de las proposiciones referidas a los números con las situaciones que enfrenta un docente a la hora de demostrar un teorema; la propuesta que en este documento se hace es vincular a las demostraciones analíticas algébricas de los números reales con las propiedades de las figuras geométricas. De esta manera se pretende que, al estar las demostraciones asociadas a representaciones gráficas geométricas visuales, el interés de los estudiantes por desarrollar las mismas e incrementar el pensamiento lógico formal, no sea algo aburrido y sin importancia.

Esta estrategia, como sugerencia para las clases de Matemáticas, está dirigida para que el estudiante no solo comprenda y disfrute de la demostración del teorema, sino que acepte la verdad y validez del mismo frente a su escepticismo o duda. También se pretende que, una asignatura como la Geometría, sea protagonista en la enseñanza de la Matemática, así como es el Álgebra de los Reales, para evidenciar esa unidad intrínseca que existe entre las diferentes partes de la Matemática.

Si el Álgebra estudia el número en la forma más general posible; el Álgebra, es la generalización de la Aritmética, concebida esta última como una de las asignaturas iniciales de la Matemática que se ocupa del estudio de las propiedades y operaciones con los números. La Aritmética junto con la Geometría fueron las dos ramas originales de la Matemática porque son las que tuvieron que enfrentase a los problemas iniciales que tuvo la misma como fueron los de contar y medir.

El protagonismo que la Geometría debe siempre tener no ha ido a la par con el Álgebra. En el nivel medio, casi siempre ha estado relegada, no solo por su escaza enseñanza sino por la mal planteada forma

de impartirla. Es decir, limitarse al memorismo de propiedades y fórmulas. La Geometría es una asignatura muy carismática, capaz de despertar un alto interés en los estudiantes porque permite abordar problemas con un carácter lúdico e informal. La Geometría, además de permitir manipular los objetos geométricos que en todas las situaciones de la vida real aparecen, se interrelaciona con otros conceptos matemáticos, siendo los algebraicos un ejemplo de los mismos.

\section{Antecedentes}

Al-Khuwarizmi. Este matemático árabe, del siglo IX, en su libro "Sobre el cálculo mediante la reducción y la restauración”, resolvió geométricamente ecuaciones de segundo grado.

La solución que Al-Khuwarizmi propuso fue para encontrar solamente la raíz positiva de ecuaciones de la forma $x^{\wedge} 2+b x=c$ con $b>0$ y $c>0$, porque todavía en aquella época no se consideraban que los coeficientes de la variable de la ecuación podían ser negativos.

Para entender el proceso se va a resolver un ejemplo concreto como el siguiente:

$$
x^{\wedge} 2+2 x=8
$$

Se puede considerar que es el área de un cuadrado C1 de lado $\mathrm{x}$, y 2x el área de un rectángulo de 
lados 2 y x; o bien, cuatro veces el área de un rectángulo C2 de lados x y $2 / 4$. El primer miembro de la ecuación es por tanto la suma de las áreas de cinco figuras que se muestran a continuación.

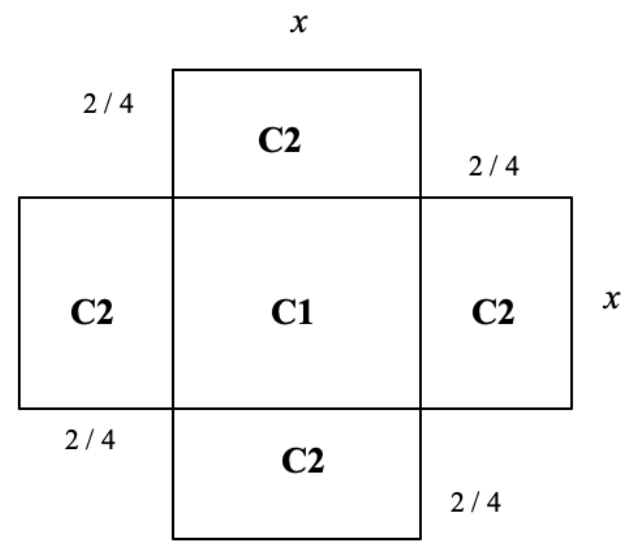

Figura. 1

La figura anterior se puede completar con cuatro cuadrados C2 en las esquinas, de tal forma que se obtiene un cuadrado de lado l, tal como se indica en la Fig. 2. Realizando la suma de las áreas de todas las figuras que componen el cuadrado de lado I resulta lo siguiente:

$$
\begin{aligned}
& \mathbf{l}^{2}=x^{2}+4\left(x * \frac{2}{4}\right)+4\left(\frac{2}{4}\right)^{2} \\
& \mathbf{l}^{2}=\left(x^{2}+2 x\right)+1 \\
& \mathbf{l}^{2}=8+1
\end{aligned}
$$

De donde se puede desprender que:

$$
\begin{array}{r}
1=3 \\
x+2\left(\frac{2}{4}\right)=3 \\
x=2
\end{array}
$$

De esta manera se ha calculado la raíz positiva $x=2$ de la ecuación planteada.

Existen métodos similares para calcular geométricamente las raíces de los demás tipos de ecuaciones de segundo grado introduciendo ligeras variaciones al proceso anterior. 
$\mathbf{C}$

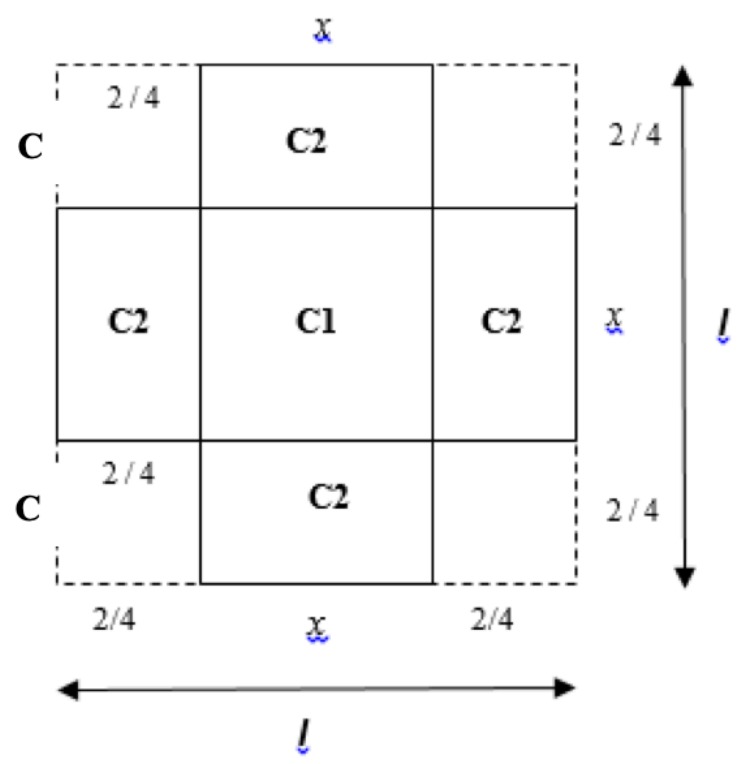

Figura. 2

Aurelio Baldor.- Baldor es un conocido autor cubano a través de su libro el Álgebra de Baldor, en el capítulo de productos y cocientes notables, hace uso de la representación gráfica geométrica para el cuadrado de la suma de dos números reales no negativos y para el producto de la suma por la resta de dos números reales también no negativos.

El cuadrado de la suma de dos números reales no negativos, en fórmula se expresa así:

$$
(a+b)^{2}=a^{2}+2 a b+b^{2}
$$

La representación geométrica para este caso comienza con la construcción de un cuadrado de lado cuyo valor es a, de tal forma que el área para este cuadrado sea $a^{\wedge} 2$. Para el área $b^{\wedge} 2$, se construye un cuadrado de lado b y para el área $2 \mathrm{ab}$, se trazan dos rectángulos de lados a $y \mathrm{~b}$. 


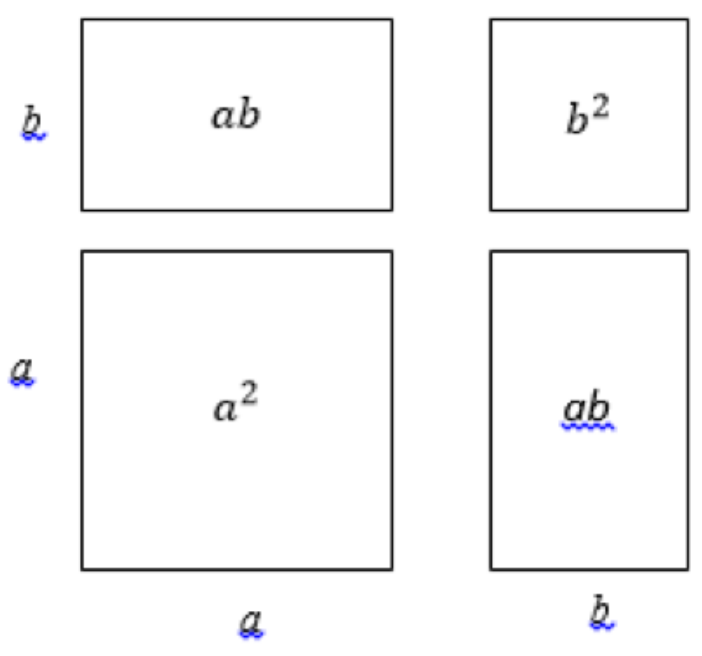

Figura. 3

Las figuras así construidas son dispuestas de tal forma que se pueda armar un cuadrado de lado $\mathrm{a}+\mathrm{b}$, cuya área $(\mathrm{a}+\mathrm{b})^{\wedge} 2$ se puede calcular sumando las áreas de los dos cuadrados y de los dos rectángulos

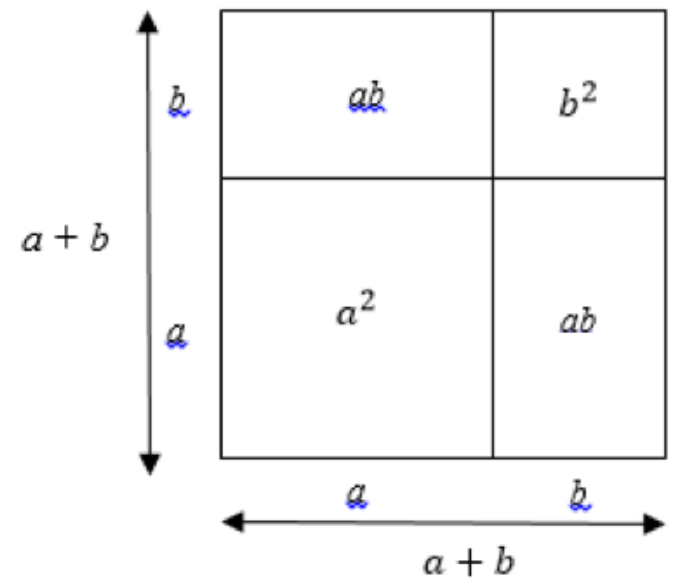

Figura. 4

La fórmula para el producto de la suma y resta de dos números reales no negativos es:

$$
(a+b)(a-b)=a^{2}-b^{2}
$$


La representación geométrica comienza, como en el caso anterior, con la construcción de un cuadrado de lado de valor a para que su área sea. Para el área , también se construye un cuadrado de lado b. El valor de a se toma mayor al valor de b.

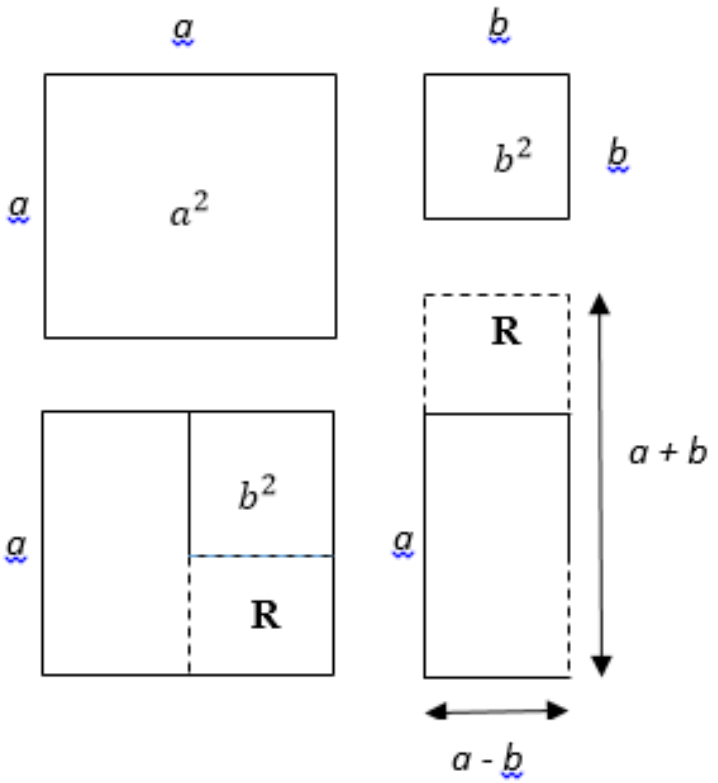

Figura. 5

Al área del cuadrado de lado a se le quita el área del cuadrado de lado $b$. Trazando una línea de puntos se obtiene un rectángulo $\mathrm{R}$ de lados $\mathrm{b}$ y $\mathrm{a}-\mathrm{b}$. Este rectángulo $\mathrm{R}$ se traslada de tal forma de llegar a obtener otro rectángulo de lados $\mathrm{a}+\mathrm{b}$ y $\mathrm{a}-b$. Al calcular el área de este último rectángulo se obtiene $(a+b)(a-b)$.

1.1.3. Pedro Miguel González Urbaneja.- Este profesor de Matemáticas de la Politécnica de Cataluña y en la Universidad de Barcelona, usa representaciones gráficas geométricas para visualizar sumatorias y series. Este matemático español es miembro de dos grupos en la red Facebook. Un grupo llamado Aprende Matemáticas; y otro, Ilamado Matemáticos. En estos grupos, Miguel González comparte y presenta, entre muchos otros temas que hacen relación con Matemáticas, las representaciones geométricas de algunas sumatorias y series que llevan por título "Prueba sin Palabras". El autor solo presenta la imagen, sin ningún comentario, dejando a la imaginación del lector la interpretación. Desde luego, esta interpretación conduce aceptar la validez de la fórmula expuesta porque la proposición deducida, tras analizar la gráfica, confirma la veracidad de la misma. A continuación, se exponen dos imágenes extraídas de los grupos de Facebook de los cuales el matemático González Urbaneja es miembro. 


\section{Primera imagen}

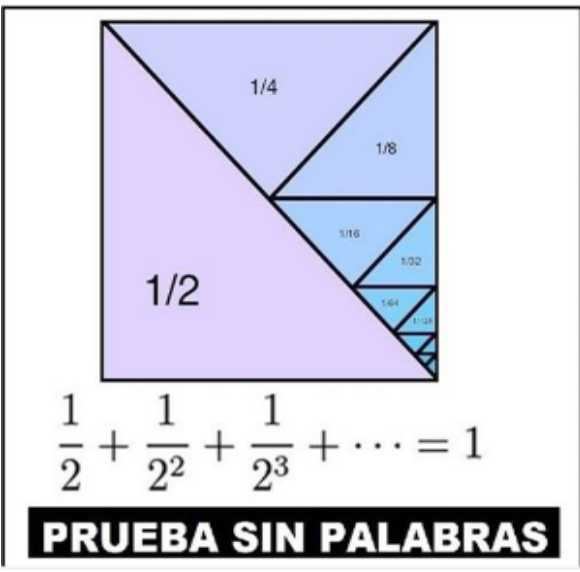

Figura. 6

En esta imagen se puede visualizar que a partir de un cuadrado de lado 1, al que se le ha trazado una de sus diagonales, se van obteniendo triángulos rectángulos isósceles al trazar la altura relativa a la hipotenusa. Cada nuevo triángulo que se forma tiene un área igual a la mitad de su respectivo anterior. Si el proceso continúa y se analizan los resultados de estas áreas, se desprende que dichos valores son los términos de una progresión geométrica decreciente infinita de razón $\mathrm{r}=1 / 2$. A saber:

\begin{tabular}{|c|c|c|c|c|c|c|}
\hline$a_{1}$ & $a_{2}$ & $a_{3}$ & $a_{4}$ & $a_{5}$ & -- & $a_{n}$ \\
\hline $1 / 2$ & $1 / 4$ & $1 / 8$ & $1 / 16$ & $1 / 32$ & -- & -- \\
& & & & & - & \\
\hline $1 / 2^{1}$ & $1 / 2^{2}$ & $1 / 2^{3}$ & $1 / 2^{4}$ & $1 / 2^{5}$ & -- & $1 / 2^{\mathrm{n}}$ \\
& & & & & - & \\
\hline
\end{tabular}

La suma de las potencias naturales de 1/2, usando el símbolo de sumatoria, se expresa así:

$$
\frac{1}{2^{1}}+\frac{1}{2^{2}}+\frac{1}{2^{3}}+\frac{1}{2^{4}}+\frac{1}{2^{5}}+\cdots+\frac{1}{2^{n}}=\sum_{n=1}^{n} \frac{1}{2^{n}}
$$


Si al número de términos $\mathrm{n}$ de esta suma se hace crecer sin límite; es decir, hasta el infinito, resulta una serie geométrica convergente que tiende al valor 1 como límite; es decir, al valor del área del cuadrado. La evidencia de lo que se acaba de enunciar es validada en forma visual por el gráfico de la Fig. 5.

$$
\sum_{n=1}^{\infty} \frac{1}{2^{n}}=1
$$

La suma $S$ de los términos de una progresión geométrica decreciente infinita puede calcularse usando la fórmula:

$$
\mathrm{S}=\frac{a_{1}}{1-r}
$$

Si en esta fórmula se reemplazan los valores correspondientes a_1 $1=1 / 2$ y $r=1 / 2$, resulta lo siguiente:

$$
S=\frac{1 / 2}{1-1 / 2}
$$

$$
S=1
$$

Por tanto, $\sum_{n=1}^{\infty} \frac{1}{2^{n}}=\mathrm{S}=1$

De esta manera se comprueba analíticamente que la deducción obtenida a partir de la gráfica es correcta. 


\section{Segunda imagen}

$$
\begin{aligned}
& \frac{1}{3}+\frac{1}{9}+\frac{1}{27}+\frac{1}{81}+\cdots+\frac{1}{3^{\mathrm{k}}}+\cdots=\frac{1}{2} \\
& \sum_{k=1}^{+\infty} \frac{1}{3^{k}}=\underset{1 / 3}{\frac{1 / 9}{1 / 27}}=\frac{1}{2} \\
& \text { PRUEBA SIN PALABRAS }
\end{aligned}
$$

Figura. 7

En esta segunda imagen también se tiene un cuadrado de lado 1, al que se le ha trazado una de sus diagonales. A este cuadrado, mediante dos segmentos de recta horizontales, se le ha dividido en tres partes iguales, o lo que es lo mismo, en tres rectángulos congruentes con un área igual a la tercera parte del área del cuadrado; que para facilitar su interpretación se han pintado en verde y en rosado.

Al rectángulo ubicado en la mitad del cuadrado, también se le ha dividido en tres partes iguales al trazar dos segmentos de recta verticales que pasan por los puntos de intersección entre los segmentos horizontales y la diagonal del cuadrado. Esto es así porque el cuadrado no solo está dividido en tres partes iguales por los dos segmentos de recta horizontales sino también por dos segmentos de recta verticales. Las figuras que resultan al interior del rectángulo dividido son tres cuadrados congruentes cuya área es un tercio de la tercera parte del área del cuadrado; es decir, $1 / 9$.

Si el proceso se vuelve aplicar a todos los cuadrado ubicados en la parte central de la figura, usando la misma diagonal y segmentos de recta horizontales y verticales, en forma alternada, los valores de las áreas que se van a obtener corresponden, como en la primera imagen, a una progresión geométrica decreciente infinita de razón 1/3. La serie geométrica que de ella se desprende, tal como se puede visualizar, converge al valor de $1 / 2$.

$$
\frac{1}{3^{1}}+\frac{1}{3^{2}}+\frac{1}{3^{3}}+\frac{1}{3^{4}}+\cdots+\frac{1}{3^{k}}+\cdots=\sum_{k=1}^{\infty} \frac{1}{2^{k}}=\frac{1}{2}
$$

1.1.4. David Hervas, doctor en Matemáticas y profesor de la Universidad San Francisco de Quito, mientras se desarrollaba la Olimpiada de Matemática, para nivel medio, en la escuela Politécnica 
Nacional, en el año 2010, realizó una charla divulgativa en la que expuso la forma como se podía realizar la descomposición en factores de un polinomio real de segundo grado en $x$, si se usaban figuras geométricas planas como cuadrados y rectángulos acompañados de las fórmulas para calcular sus áreas. La forma que este matemático usó tiene mucha relación con la que Al-Khuwarizmi usó para calcular la raíz positiva de una ecuación de segundo grado con coeficiente reales positivos. A continuación se presentan las imágenes de aquella exposición, en la cual se tomó como ejemplo, para explicar el proceso, el polinomio dado por la fórmula de definición .

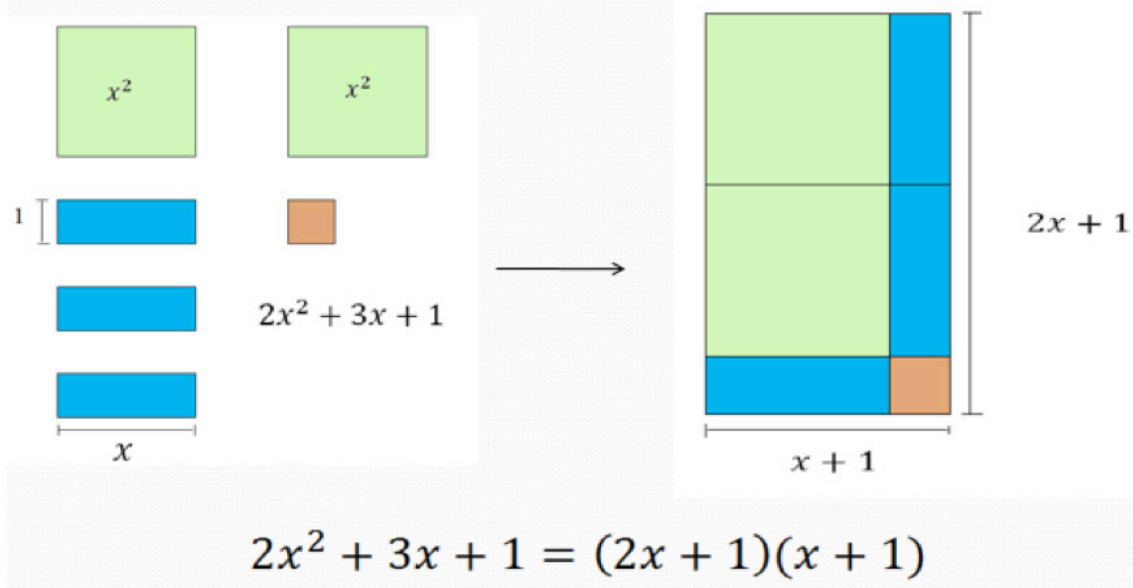

Figura. 8

En estas imágenes se pueden observar tres cuadrados, dos de lado de valor $x$, para obtener un área igual a $2 \mathrm{x}^{\wedge} 2$ y uno de lado de valor 1 , que como es obvio tiene un área igual a 1 . Hay tres rectángulos de lados x y 1 con los cuales resulta el área $3 \mathrm{x}$. Con las figuras así construidas, se arma un rectángulo, en el cual la suma de las áreas de las figuras que lo forman es igual al área calculada al multiplicar el largo $2 x+1$ por el ancho $x+1$. De esta forma el polinomio cuadrático ha sido descompuesto en el producto de dos factores de primer grado.

\section{DESARROLLO}

En el Álgebra del Conjunto de los Números Reales R, un teorema bastante conocido es el siguiente:

"La media aritmética de dos números reales no negativos a y $b$ es mayor o igual que su media geométrica".

En fórmula, el teorema se expresaría así:

$$
\frac{a+b}{2} \geq \sqrt{a b}
$$




\section{Representación Escrita en Forma Analítica de la Demostración del Teorema.}

Antes de hacer una representación escrita analítica del teorema, partiendo de las premisas para llegar a la tesis o conclusión, se va a invertir el razonamiento; es decir, partiendo de la tesis resulta lo siguiente:

$$
\begin{aligned}
& \operatorname{Si} \frac{a+b}{2} \geq \sqrt{a b}, \text { se tiene }(a+b)^{2} \geq(2 \sqrt{a b})^{2}, a^{2}+2 a b+b^{2} \geq 4 a b, a^{2}-2 a b+b^{2} \geq 0, \\
& (a-b)^{2} \geq 0 \text {. Esta última expresión es verdadera para cualquier valor real de } a \text { y b. }
\end{aligned}
$$

A partir de la premisa, que sabemos es verdadera, la representación escrita, en forma analítica, de

\begin{tabular}{|c|c|}
\hline Proposiciones & Justificaciones \\
\hline $\begin{array}{l}\text { 1. } \mathrm{a} \text { y } \mathrm{b} \text { son números reales no } \\
\text { negativos. }\end{array}$ & 1. Hipótesis \\
\hline 2. $(a-b)^{2} \geq 0$ & $\begin{array}{l}\text { 2. Teorema: } \\
\text { Todo número real elevado al cuadrado } \\
\text { es mayor o igual que cero. }\end{array}$ \\
\hline $\begin{array}{l}\text { 3. } a^{2}-2 a b+b^{2} \geq 0 \\
\text { 4. } a^{2}+2 a b+b^{2} \geq 4 a b\end{array}$ & $\begin{array}{l}\text { 3. Cuadrado de un binomio. } \\
\text { 4. Teorema: } \\
\text { El sentido de una desigualdad no } \\
\text { cambia si se suma un mismo número } \\
\text { real a sus dos miembros. }\end{array}$ \\
\hline 5. $(a+b)^{2} \geq(2 \sqrt{a b})^{2}$ & $\begin{array}{l}\text { 5. Identidad: Trinomio cuadrado } \\
\text { perfecto. }\end{array}$ \\
\hline 6. $a+b \geq 2 \sqrt{a b}$ & $\begin{array}{l}\text { 6. Extracción de raíces cuadradas a } \\
\text { números reales no negativos. }\end{array}$ \\
\hline 7. $\frac{a+b}{2} \geq \sqrt{a b}$ & $\begin{array}{l}\text { 7. Teorema: } \\
\text { El sentido de una desigualdad no } \\
\text { cambia si a ambos miembros se les } \\
\text { divide por un mismo número real } \\
\text { positivo. }\end{array}$ \\
\hline
\end{tabular}
la demostración del teorema sería:

Para este teorema se desarrollan tres representaciones gráficas, geométricas visuales. 


\section{Representación Geométrica 1.}

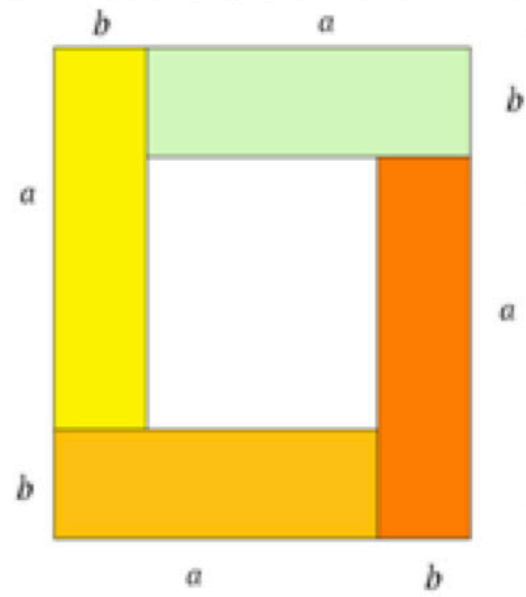

En esta gráfica se tienen 4 rectángulos cuyos lados miden los números reales no negativos a y b. La disposición de los 4 rectángulos hace que se forme un cuadrado de lado a $+b$. Luego, el área de este cuadrado es $(a+b)^{2}$.

En la figura se aprecia el que el área del cuadrado es mayor que el área 4 ab de los 4 rectángulos. Para que el área de los 4 rectángulos sea igual al área del cuadrado hace falta sumarle el área del cuadrado interior $(a-b)^{2}$.

$$
\begin{gathered}
(a+b)^{2} \geq 4 a b \\
a+b \geq 2 \sqrt{a b} \\
\frac{a+b}{2} \geq \sqrt{a b}
\end{gathered}
$$




\section{Representación Geométrica 2.}

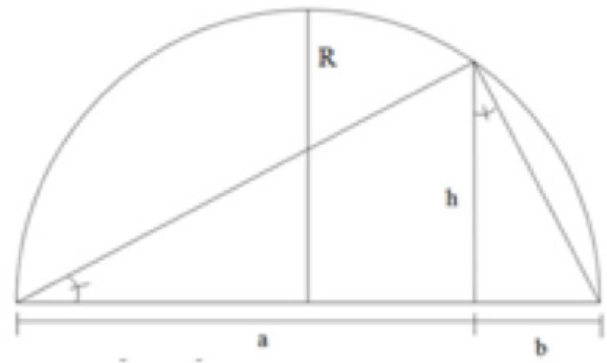

$\mathrm{C}$

Figura. 10

En esta otra representación se toma en cuenta dos teoremas de la Geometría Plana.

a. Teorema.- Todo ángulo inscrito en un semicírculo es recto.

En la gráfica, el ángulo inscrito en el semicírculo es el ángulo $A B C$ y por ende mediría $90^{\circ}$.

b. Teorema.- La altura $\mathrm{h}$ relativa a la hipotenusa de un triángulo rectángulo es media proporcional entre los segmentos a y b que determina en la hipotenusa.

$$
\begin{aligned}
\frac{h}{a} & =\frac{b}{h} \\
\mathrm{~h} & =\sqrt{a b}
\end{aligned}
$$

En la figura se visualiza que la altura $\mathrm{h}$ es menor o igual que el radio $\mathrm{R}$ del semicírculo. También que $\mathrm{R}$ es igual a la mitad del diámetro $\mathrm{a}+\mathrm{b}$. Por tanto:

$$
\begin{array}{r}
\mathrm{R} \geq h \\
\frac{a+b}{2} \geq \sqrt{a b}
\end{array}
$$




\subsection{Representación Geométrica 3}

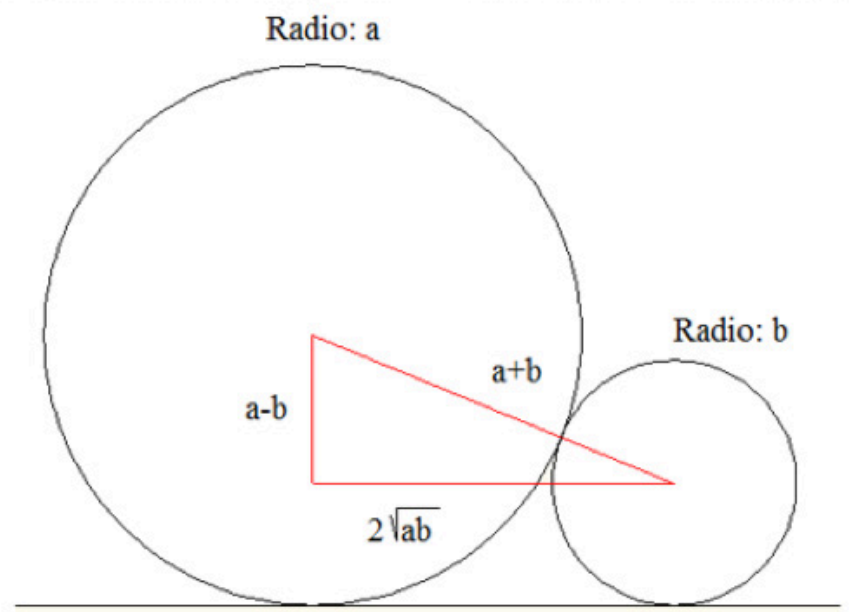

Figura 11

En la gráfica se tienen dos círculos tangentes de radios a y b. Al formarse el triángulo rectángulo se observa que la hipotenusa es la suma $\mathrm{a}+\mathrm{b}$ de los dos radios y uno de los catetos la diferencia $\mathrm{a}-$ $\mathrm{b}$ entre estos. Al calcularse el cateto faltante, aplicando el teorema de Pitágoras, se obtiene como resultado $2 \sqrt{\mathrm{ab}}$. Del triángulo rectángulo, como cualquier cateto es menor que la hipotenusa, por ser este lado siempre mayor que cualquier otro, se obtiene la expresión para la desigualdad.

$$
\begin{gathered}
a+b \geq 2 \sqrt{a b} \\
\frac{a+b}{2} \geq \sqrt{a b}
\end{gathered}
$$

\subsection{Aplicación}

2.5.1. A continuación, se presenta un problema donde se puede apreciar como la representación geométrica, a través de un gráfico, ayudaría para la demostración analítica de la proposición que se plantea.

Enunciado.- Sean $a_{1}, A_{1}, a_{2}, A_{2}, a_{3}, A_{3}$ números reales positivos tales que $a_{i}+A_{i}=k$, donde $\mathrm{k}$ es una constante, Sáenz (2010). Demostrar que:

$$
a_{1} A_{2}+a_{2} A_{3}+a_{3} A_{1}<k^{2}
$$




\section{Representación Geométrica}

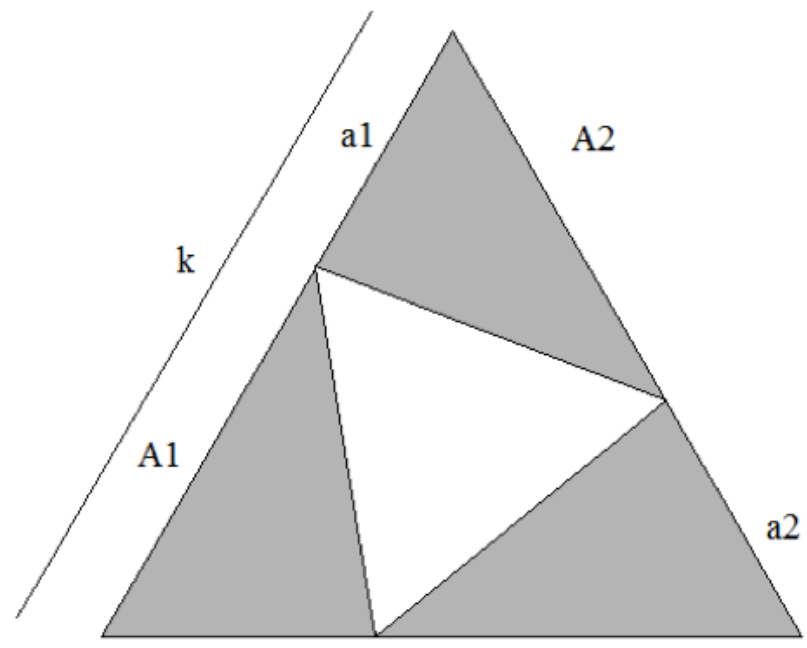

a3

A3

Figura. 12

Como $a_{-} \mathrm{i}+\mathrm{A}_{-} \mathrm{i}=\mathrm{k}$, el gráfico geométrico que encaja es el de un triángulo equilátero, cuyo lado de valor $\mathrm{k}$, es la suma de dos segmentos a_i y A_i. Además, como también se tiene a_1 A_2+a_2 A_3+ a_3 A_1, los segmentos que forman el lado del triángulo equilátero, han sido ubicados de tal forma que al calcularse el área de los triángulos escalenos sombreados, puedan obtenerse los productos a_1 A_2,a_2 A_3 y a_3 A_1. Por tanto:

Área de la región sombreada:

$$
\begin{aligned}
& \quad \frac{1}{2} \operatorname{sen}\left(60^{\circ}\right)\left(a_{1} A_{2}+a_{2} A_{3}+a_{3} A_{1}\right)=\frac{\sqrt{3}}{4}\left(a_{1} A_{2}+\right. \\
& \left.a_{2} A_{3}+a_{3} A_{1}\right)
\end{aligned}
$$

Área del triángulo equilátero: $\quad \frac{\sqrt{3}}{4} \mathrm{k}^{2}$

Como el área de la región sombreada es menor que el área del triángulo equilátero, resulta lo siguiente:

$$
\frac{\sqrt{3}}{4}\left(a_{1} A_{2}+a_{2} A_{3}+a_{3} A_{1}\right)<\frac{\sqrt{3}}{4} \mathrm{k}^{2}
$$


De donde se obtiene que:

$$
a_{1} A_{2}+a_{2} A_{3}+a_{3} A_{1}<k^{2}
$$

2.5.2. La representación gráfica geométrica visual también permite, mediante simple observación, deducir proposiciones. Tal es el ejemplo que se muestra.

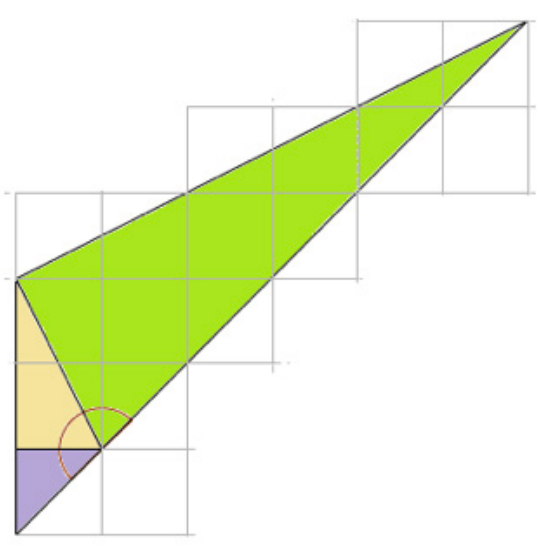

Figura. 13

$$
\tan ^{-1}(1)+\tan ^{-1}(2)+\tan ^{-1}(3)=\pi
$$

Explicación.- Fijemos nuestra atención en el vértice común a los tres triángulos rectángulos. Este vértice pertenece al triángulo rectángulo que es isósceles por tener dos catetos de igual valor. Luego, el valor del ángulo agudo asociado a este vértice para este triángulo, calculado por Trigonometría, es $\tan ^{\wedge}(-1)(1)$; es decir, $45^{\circ}$.

Si nuevamente tomamos en consideración el mismo vértice, pero ahora fijándonos en el triángulo rectángulo que tiene un cateto de valor doble que el otro, se puede desprender que el valor del ángulo agudo asociado a este vértice para este triángulo, calculado por Trigonometría, es $\tan ^{\wedge}(-1)$ (2); es decir, $63,435^{\circ}$.

Por último, observando el mismo vértice pero el triángulo rectángulo que tiene un cateto que es el triple del otro, resulta que el valor del ángulo agudo asociado a este vértice para este triángulo, calculado por Trigonometría, es $\tan ^{\wedge}(-1)$ (3); es decir, $71,565^{\circ}$.

Como el vértice analizado es común a tres ángulos consecutivos que forman un ángulo llano o de lados colineales, la suma de las medidas de los valores de estos tres ángulos es $\pi$; o lo que es lo mismo, $180^{\circ}=45^{\circ}+63,435^{\circ}+71,565^{\circ}$. 


\section{ANÁLISIS DE RESULTADOS}

Las representaciones gráficas geométricas visuales, no solo han mostrado la evidencia del teorema tratado, sino también esa íntima relación entre el Álgebra de los Números Reales y la Geometría Plana, dos materias de una misma ciencia. Esta relación da profundidad al conocimiento y eleva la capacidad de demostración.

En el Algebra de Números Reales las demostraciones no siempre incluyen un gráfico; y todavía, geométrico. Una demostración así desarrollada no permite que muchos estudiantes alcancen una plena compresión de lo que se está tratando y hace a la Matemática demasiado abstracta e incomprensible. En los estudiantes universitarios de los niveles básicos, quienes no están familiarizados con el aprendizaje deductivo inferencial, es donde más se acentúa este problema y por ende ven a la Matemática poco agradable y difícil de aprenderla, Benalcázar (2007).

Ante la dificultad del aprendizaje de la Matemática en forma deductiva inferencial, porque no se enseña a buscar los pasos de la demostración con el empleo de procedimientos heurísticos; es el memorismo y mecanicismo los que priman. Si la Matemática es fruto del raciocinio lógico formal del ser humano, memorismo y mecanicismo se deben erradicar, tanto en la enseñanza como en el aprendizaje de Matemáticas, porque desvirtúan el alma y esencia de esta ciencia exacta.

A lo largo de mi carrera docente, de más de veinte años en las universidades de mi país, he constatado que en los primeros años los niveles de deserción y pérdida son muy altos. Una de las asignaturas que más dificultad presenta es la Matemática. La forma de enseñanza y aprendizaje de la Matemática a nivel superior, evidencia diferencias marcadas de aquella que es impartida en el nivel medio. Los estudiantes deben estar acostumbrados a que en una clase de Matemáticas realizar demostraciones es tan corriente como resolver problemas o desarrollar ejercicios.

\section{CONCLUSIONES}

Un estudiante desarrolla su pensamiento cuando penetra en la esencia del fenómeno; no cuando repite muchas veces un mismo proceso. Relacionar formas para demostrar la validez de una afirmación es una manera de convencer al escéptico.

Las demostraciones tienen su importancia, cuando responden a las dudas de los estudiantes, probando aquello que no es obvio, que no pueden "visualizar".

Una representación gráfica geométrica visual garantiza una adecuada secuencia en el pensamiento a la hora de demostrar y da solidez a las destrezas que en torno a ella se crean y desarrollan.

Los años básicos de las universidades deben constituir un puente para que el bachiller, novato universitario, pase de la educación media a la superior, sin mayor dificultad. Por tanto, es aquí donde la forma de la enseñanza y aprendizaje de la Matemática debe ser fortalecida para que el estudiante mire a esta ciencia con un grado de apego y respeto. Ante este hecho, a la forma 
analítica formal de demostración de un teorema se debe agregar una representación gráfica geométrica visual. Es decir, incorporar las figuras geométricas planas y sus propiedades en la demostración de los teoremas del Algebra del Campo de los Números Reales. De esta manera se espera que el estudiante se motive y retire de su pensamiento el temor y las dificultades que trae el aprender esta ciencia noble pero dura en su dominio y comprensión.

Cuando hay muchas dificultades para realizar una demostración analítica, realizar un gráfico geométrico es una sugerencia a tomar en cuenta para facilitar el proceso, sobre todo cuando se está en las etapas iniciales para aprender a demostrar.

La importancia de enseñar Matemática en la forma correcta ayuda a los estudiantes en su capacidad de raciocinio, observación, análisis y síntesis, en definitiva; desarrolla las formas de pensamiento de quien la estudia o enseña.

Corregir la forma de enseñanza y aprendizaje de la Matemática va a ayudar a muchos estudiantes no solo a no reprobar los cursos, sino también a cambiar las estructuras mentales y concepciones equívocas que traen con respecto a esta ciencia. 


\section{REFERENCIAS BIBLIOGRÁFICAS}

ALBA T., El Hombre que Calculaba. 2da ed. Gráficas Modernas. Bogotá.

ANTONOV N., VYGOSDSKY M., et al. 1000 Problemas de Aritmética, Álgebra, Geometría y Trigonometría. Paraninfo. Madrid. 2015.

BALDOR A., Algebra. Edime Organización Gráfica, S.A. Madrid. 1976.

BARNETH R., URIBE J. Álgebra y Geometría. 4ta ed. Vol 1. McGraw-Hill. Bogotá. 2015.

BARNETH R., URIBE J. Álgebra y Geometría. 4ta ed. Vol 2. McGraw-Hill. Bogotá. 2015.

BENALCÁZAR H. Matemática para el Bachillerato. Tomo 1. El Gran Libro. Quito. 2007.

BRUÑO. Álgebra Superior. 13va ed. Madrid. 2016.

CALCACHE G., ROSERO T., et al. Geometría Plana y del Espacio. Escuela Politécnica Nacional. Quito. 2011.

DIAZ J., GÓMEZ B., Didáctica de la Matemática. Síntesis. Madrid. 2014.

GALINDO E., Geometría. Centro de Matemática de la Universidad Central. Quito. 2006.

MURRAY R. Álgebra Superior. McGraw-Hill. Cali. 2015.

OLIVEROS E., Método de la Enseñanza de la Matemática. Santillana S. A. Quito. 2002

PROAÑO R., Lógica Conjuntos Estructuras. 9na ed. Librería Cima. Quito. 2010.

SÁENZ R. Fundamentos de Matemática. Centro de Matemática de la Universidad Central. Quito. 2010. 